\title{
Gastric cancer in Mexico
}

\author{
Luis F. OÑATE-OcaÑa
}

Gastroenterology Department, Surgery Division, National Cancer Institute, San Fernando 22, México D.F. 14000, México

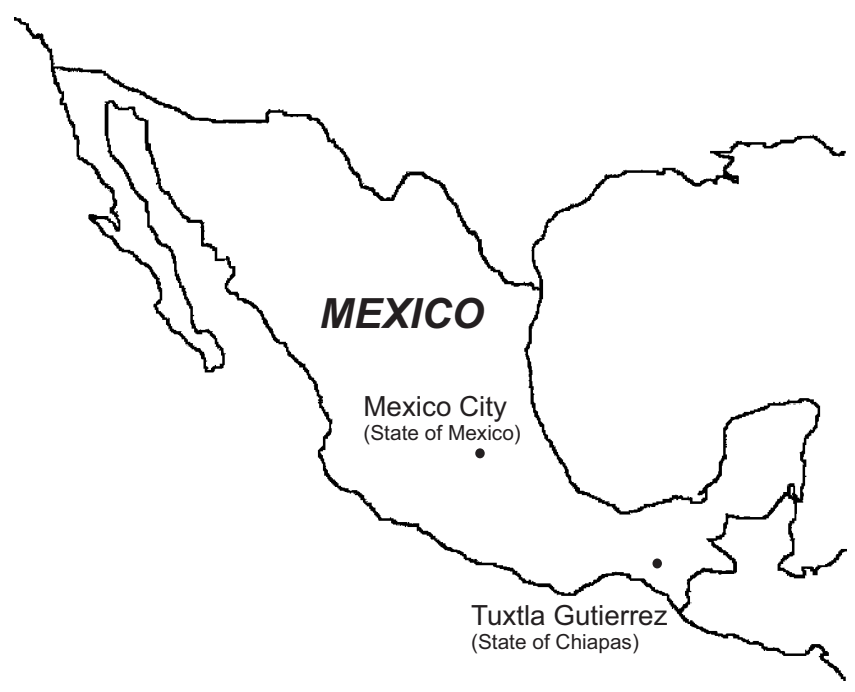

Most cancer epidemiology statistics in Mexico are extracted from the Histopathologic Register of Neoplasia in Mexico, which is the most complete register and the only one with national coverage [1]. This tumor register is based on the information generated by 930 pathologists and hematologists from 671 private and public hospitals in the country.

Based on this register, gastric cancer (GC) is the most frequent gastrointestinal malignant neoplasm in Mexico [1], and it is the second cause of cancer-related mortality after lung cancer $[1,2]$. In contrast to the declining incidence and mortality associated with this tumor in most countries, GC mortality in Mexico has been reported to be steady during the past three decades (Fig. 1) [2]. The reason for this could be increasing efficiency in clinical diagnosis and in the reporting of new cases.

Offprint requests to: L. F. Oñate-Ocaña

Received: June 11, 2001 / Accepted: July 27, 2001
Wide variations in GC incidence have been reported worldwide, and Mexico is considered a low-risk area. The crude GC-associated mortality rate is 5.1 per 100000 inhabitants [2]. However, a GC cluster has been reported in the highlands of Chiapas, in a mountainous region close to the Guatemalan border [3]. So, important differences in the country have been found, which possibly reflect differences in diet habits and in social and economic factors. Chiapas has the highest GCassociated mortality rate $\left(6.4 \times 10^{4}\right)$ in the country; in Mexico City, the risk is medium $\left(4.5 \times 10^{4}\right)$, and in Mexico State it is low $\left(2.5 \times 10^{4}\right)$.

Dietary factors related to GC have not been studied extensively in Mexico. However, specific features of the lifestyle that could be related to GC have been identified. Chili pepper is a traditional product in the Mexican diet, and some studies suggest that the hot-tasting component of Chili pepper, capsaicin, may be carcinogenic.

A case-control study performed in Mexico City suggests that Chili pepper consumers were at a higher risk of GC than nonconsumers. There was a highly significant trend of increasing risk with increasing selfrated level of consumption. Multivariable adjustment increased the magnitude of the Chili pepper-gastric cancer association. Chili pepper consumption may be a strong risk factor for gastric cancer, but further studies are needed to prove causality [4].

Saturated fat and cholesterol consumption have been identified as a major risk factor for intestinal-type GC in Mexico [5]. A reduction in the risk of developing GC has been associated with the intake of polyunsaturated fat, fiber, and vitamin $\mathrm{E}$; this effect was independent of the histological type of the tumor (intestinal or diffuse) [5]. These findings are consistent with many studies around the world that indicate important roles for salt, processed meat, and fruit and vegetable consumption in GC risk.

Gastric infection by Helicobacter pylori is present in $80 \%$ of the adult population in Mexico and is acquired 


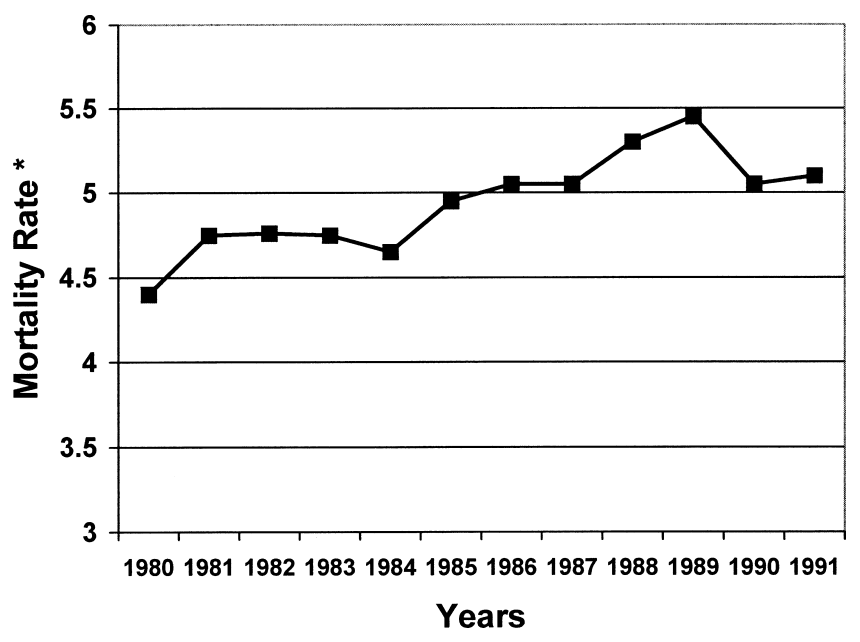

Fig. 1. Time trend for gastric cancer-associated mortality rates in Mexico from 1980 to 1991. Asterisk, mortality rates per 100000 inhabitants

in the early years of life [6]. Worldwide seroepidemiological studies on the association of $\mathrm{CagA}^{+}$antibody prevalence and GC have yielded conflicting results. In Mexico, gastric infection with $\mathrm{H}$. pylori and $\mathrm{Cag} A^{+}$ seropositivity have been reported to be significantly associated with an increased risk of mortality due to GC [6].

In the southern state of Chiapas, with the highest incidence of GC reported in Mexico, an $85.7 \%$ prevalence of $H$. pylori infection has been found [7]. This study revealed a strong association between $H$. pylori infection, determined by biopsy, with gastric atrophy, intestinal metaplasia, dysplasia, and GC [7].

Gastric Epstein-Barr virus (EBV) infection has also been implicated in the genesis of gastric carcinoma. In a Mexican study, EBV-encoded small nonpolyadenylated RNA (EBER) in-situ hybridization was performed on paraffin-embedded tissue sections of gastric tumors. Eleven (8.15\%) of 135 Mexican patients with GC were EBER-1-positive, and 5 had lymphoepithelioma-like carcinomas [8].

No specific Laurén histological type of GC predominates in Mexico. In a study of 220 cases of GC, intestinal type was found in $44.5 \%$ and diffuse type in $43.3 \%$; the remaining $12.3 \%$ corresponded to mixed type and indeterminate tumors [9], even when intestinal type predominated in high-risk areas such as Chiapas [3]. In another study, a predominance of diffuse type was observed in the young age group, and the global intestinal/ diffuse ratio was $1: 2$, with a trend to increase with age [10]. The distribution of these histological types did not vary significantly according to socioeconomic status [9].

The proportion of distal and proximal tumors in the tumor register of the National Cancer Institute has been

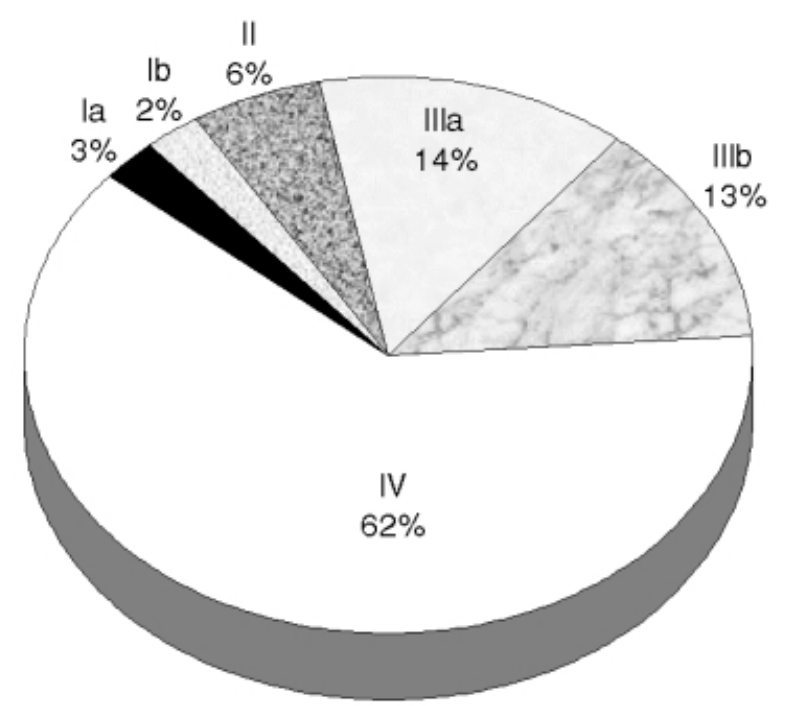

Fig. 2. Relative frequency of TNM stages $(n=793$ patients treated at the National Cancer Institute Hospital in Mexico City; modified from Oñate-Ocaña et al. ${ }^{12}$ [Rev Gastroenterol Mex 1999;64:114-21.]; with permission)

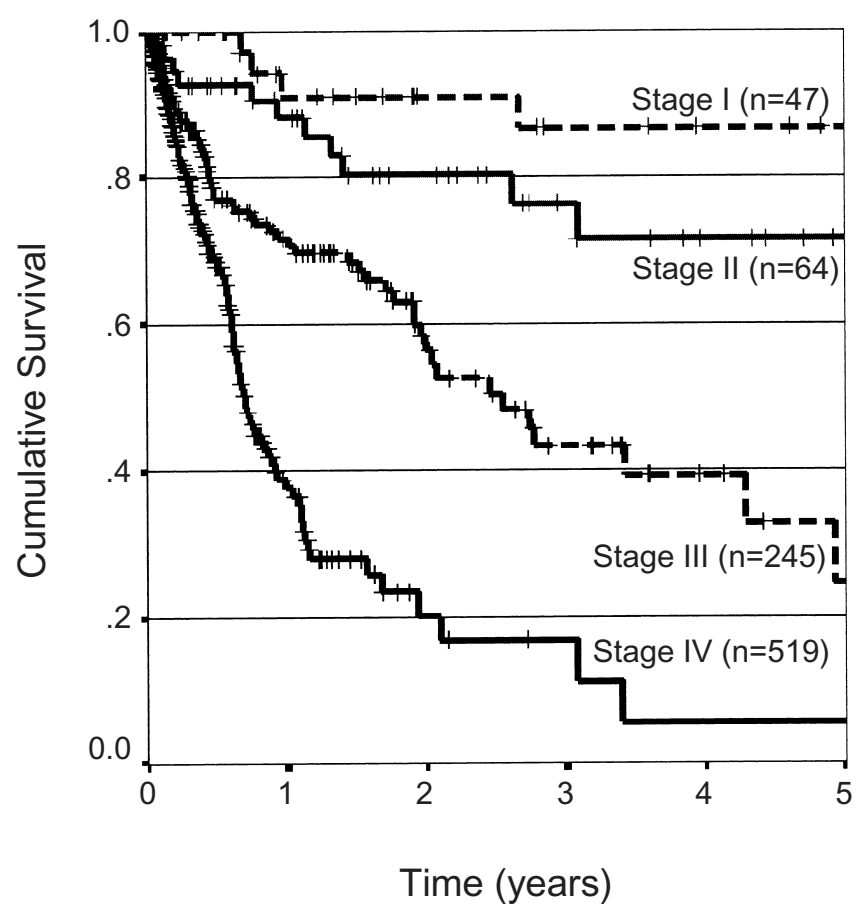

Fig. 3. Survival curves by TNM stage $(n=875$ patients with gastric carcinoma treated at the National Cancer Institute Hospital in Mexico City from January 1987 to June 2000)

fairly constant in the past 14 years. Excluding tumors invading the whole stomach, 465 patients $(66.5 \%)$ had distal tumors and 234 (33.5\%) had proximal tumors.

In Mexico, early GC is found with very low frequency. In a retrospective cohort study of 834 patients 
with GC treated at the National Cancer Institute in Mexico City, only 21 presented with early GC [11]. This represents $2.3 \%$ of all stages. None presented with lymph node metastases at the time of surgical resection, and 2 patients presented with recurrence. The 5-year survival rate of this small cohort was $84 \%$. An explanation for this extremely low proportion is selection bias. Our institute is a third-level referral hospital that specializes in unresectable cases, so the stage distribution is marked by a high proportion of far-advanced cases [12]. The relative frequency of TNM stages is depicted in Fig. 2, and survival curves by TNM stage are shown in Fig. 3.

Subtotal or total gastrectomy with D1 lymph node dissection (LND), following Western standards, is the preferred resection procedure for gastric cancer in most hospitals in Mexico. However, Japanese-style D2 LND has been used since 1985 at the National Cancer Institute in Mexico City, with promising results. In a retrospective study, D2 LND-associated surgical morbidity was similar to that of D1 LND, and a survival benefit for patients treated with D2 LND was found by multivariate analysis with adjustment for the major prognostic and confounding factors [13]. Possibly, in terms of ethnic aspects, Mexican patients are more comparable to Japanese than to North Americans or Europeans.

Adjuvant chemotherapy is not used routinely in Mexico after complete (R0) surgical resection. When it is used, the preferred combination chemotherapy is fluouracil, doxorubicin and methotrexate (FAMtx) or cisplatin, leucovorin and fluouracil (PLF) and it is administered as palliative treatment in patients with faradvanced disease.

Recently, we used the P-ELF combination (cisplatin, etoposide, leucovorin, 5-fluorouracil) as neoadjuvant chemotherapy in patients with unresectable GC. This combination chemotherapy permitted $17.5 \%$ resectability after 2.9 courses of treatment per patient [14].

In conclusion, $\mathrm{GC}$ is an important health problem in Mexico. In the coming decades, education programs and clinical research must be directed toward methods for diagnosing $\mathrm{GC}$ at early stages, because this is the only significant way to reduce mortality.

\section{References}

1. Secretaría de Salud, Asociación Mexicana de Patólogos, A.C. Instituto Nacional de Cancerología. Estómago. In: Secretaría de Salud, Ed. Compendio del Registro Histopatológico de Neoplasias Malignas en México. Ciudad de México, México: Dirección General de Epidemiología, Secretaría de Salud; 1997.

2. Secretaría de Salud. Mortalidad 1995. Ciudad de México, México: Dirección General de Epidemiología, Secretaría de Salud; 1996.

3. Halperin DC, Mohar A. Stomach cancer cluster in Mexico. Lancet 1988;1:1055.

4. Lopez-Carrillo L, Hernandez Avila M, Dubrow R. Chili pepper consumption and gastric cancer in Mexico: a case-control study. Am J Epidemiol 1994;139:263-71.

5. Lopez-Carrillo L, Lopez-Cervantes M, Ward MH, BravoAlvarado J, Ramirez-Espitia A. Nutrient intake and gastric cancer in Mexico. Int J Cancer 1999;83:601-5.

6. Torres J, Perez-Perez GI, Leal-Herrera Y, Munoz O. Infection with $\mathrm{CagA}^{+}$Helicobacter pylori strains as a possible predictor of risk in the development of gastric adenocarcinoma in Mexico. Int J Cancer 1998;78:298-300.

7. Guarner J, Mohar A, Parsonnet J, Halperin D. The association of Helicobacter pylori with gastric cancer and preneoplastic gastric lesions in Chiapas, Mexico. Cancer 1993;71:297-301.

8. Herrera-Goepfert R, Reyes E, Hernandez-Avila M, Mohar A, Shinkura R, Fujiyama C, et al. Epstein-Barr virus-associated gastric carcinoma in Mexico: analysis of 135 consecutive gastrectomies in two hospitals. Mod Pathol 1999;12:873-8.

9. Lopez-Carrillo L, Vega-Ramos B, Costa-Dias R, Rascon-Pacheco RA. Histological types of gastric cancer in Mexico. Int $\mathbf{J}$ Epidemiol 1997;26:1166-71.

10. Mohar A, Suchil-Bernal L, Hernandez-Guerrero A, PodolskyRapoport I, Herrera-Goepfert R, Mora-Tiscareno A, AielloCrocifoglio V. Intestinal type: diffuse type ratio of gastric carcinoma in a Mexican population. J Exp Clin Cancer Res 1997;16:189-94.

11. Oñate-Ocaña LF, Cortés-Cárdenas $\mathrm{S}$, Herrera-Goepfert $\mathrm{R}$, Aiello-Crocifoglio V, Mondragón-Sánchez R, Ruis-Molina JM. Early gastric cancer: analysis of 21 cases. Rev Gastroenterol Mex 2001;66:14-21.

12. Oñate-Ocaña LF, Aiello-Crocifoglio V, Mondragon Sanchez R, Ruiz Molina JM, Gallardo-Rincon D. Prognostic factors in 793 cases of gastric cancer in an oncologic referral center. Rev Gastroenterol Mex 1999;64:114-21.

13. Oñate-Ocaña LF, Aiello-Crocifoglio V, Mondragón-Sánchez R, Ruiz Molina JM. Survival benefit of D2 lymphadenectomy in patients with gastric adenocarcinoma. Ann Surg Oncol 2000;7: $210-7$.

14. Gallardo-Rincón D, Oñate-Ocaña LF, Calderillo-Ruiz G. Neoadjuvant chemotherapy with P-ELF (cisplatin, etoposide, leucovorin, 5-fluorouracil) followed by radical resection in patients with initially unresectable gastric adenocarcinoma. Ann Surg Oncol 2000;7:45-50. 